\title{
Average evoked potentials and uncertainty resolution
}

\author{
EMANUEL DONCHIN, ${ }^{-N A S A-A M E S ~ R E S E A R C H ~ C E N T E R, ~}$ \\ Moffett Field, California 94035
}

Two Ss were presented with a series of near threshold flashes of light and instructed to report for each flash in which of eight different positions it appeared, and the degree to which they were certain about this judgment. Average evoked potentials to the flashes were also recorded from the occiput. A positive-going wave with latency to the peak of 250 msec appeared when the $S$ was certain about his judgment, whether or not he was correct.

Sutton and his coworkers (1967) have reported that when a stimulus resolves a S's uncertainty, a positive going wave appears in the average evoked potential (AEP) recorded from the S's scalp, with a latency to the peak of about $300 \mathrm{msec}$. Similar findings were reported by Donchin \& Cohen (1967), Chapman \& Bragdon (1964), and others. The present report describes a study of average evoked potentials to near-threshold stimuli the results of which serve to further confirm Sutton's findings.

Method. A test flash (TF) of light was obtained from a Sylvania glow modulator tube (R1131C) and presented in Maxwellian view to the right eye of the $S$. The TF was presented through a semicircular stop defining a visual angle of $1 \mathrm{deg} 22 \mathrm{~min}$ along its diameter. The stop could be rotated at random to any one of eight different positions. Four dim red lines produced by a broken cross reticle converged upon the stimulus area and provided a fixation point. The current pulse through the glow modulator was maintained at $35 \mathrm{~mA}$ throughout the experiment. The luminance thus obtained was 6000 $\mathrm{mL}$. Flash luminance was reduced with Wratten neutral density filters by 4.3 $\log$ units.

Two Ss participated in the experiment and were each run on four sessions. Each session consisted of several 120 trials series of presentations. Before each trial the experimenter rotated the TF stop to one of the eight positions. The $S$ was then instructed to press a switch triggering the TF when properly fixated, and to report the position of the TF. A choice was forced on the Ss on each trial, however they were instructed to indicate whether or not the choice was a "guess."

The EEG was recorded, using a Grass model 7 encephalograph, on FM magnetic tape (Ampex FR 1300). Electrodes were placed on the occiput (2.5 $\mathrm{cm}$ above and to the right of the inion). Recording was "monopolar" to an electrode on the left ear. Trigger pulses synchronous with the TF were also recorded on the tape. Following each trial the experimenter placed on the tape a pulse code identifying the outcome as one of four possible outcomes: (1) correct identification-S certain, (2) correct identification-S guessing, (3) incorrect-certain, (4) incorrect-guessing. Average evoked responses were obtained for each session-one for each of the four categories of trial outcome. Averages were obtained with the T.M.C. CAT.

Results. Figure 1 presents the data from one $\mathrm{S}$ for one session. ${ }^{2}$ Similar results were obtained in all other sessions. It can be seen that a large positive wave with a latency of about $250 \mathrm{msec}$ appears whenever the $S$ reports that he is "certain." It does not appear when the $S$ reports that he is guessing. The difference between "correct" and "incorrect" identifications is small and appears to be mainly in the "sharpness" of the positive wave-the "correct" trials yielding a sharper average.

Discussion. This study was originally designed in order to determine if there is a difference between AEP elicited by TFs whose positions were identified correctly and incorrectly. As such the study was a failure. Practically no difference could be observed between these AEPs. On the other hand, the degree to which the $S$ was certain about his position judgment did affect greatly the amplitude of the evoked response.

These results can be interpreted within the framework provided by Sutton's work cited above. The experimental setup is such that the $S$ is awaiting the presentation of the stimulus to resolve an uncertainty about TF position. When some event leads him to resolve this uncertainty this event elicits a positive wave with a latency of $250-300$ msec. This wave appears regardless of the specific visual stimulation and depends solely on the S's perception of the situation. Thus, when his uncertainty is not resolved and he reports a "guess," no corresponding wave is obtained.

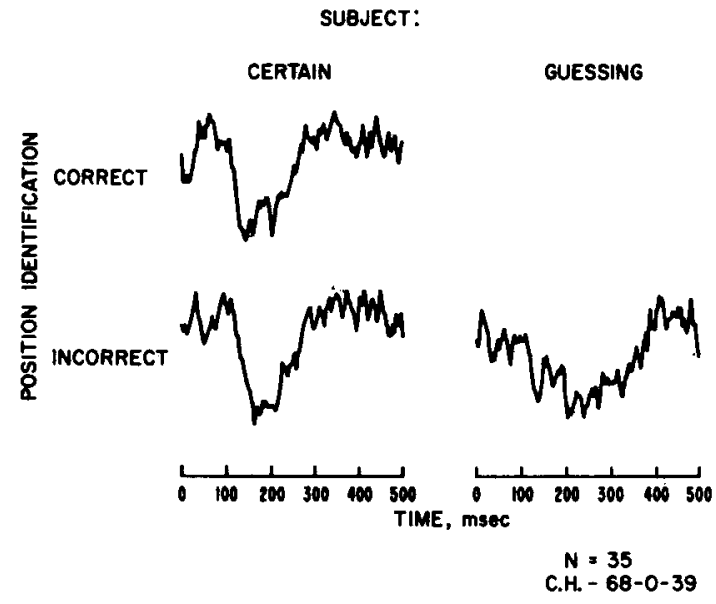

Fig. 1. Average evoked potentials for near threshold stimuli. The three records represent one series of stimuli averaged in three groups according to S's response. 0

It should be noted that at the luminance level used, the Ss rarely saw the full contour of the semicircle. What they saw was an ill-defined blurb of light. Thus the position judgments were actually based on the apparent distribution of light in the field. "Certain" judgments were probably obtained whenever this blurb of light was observed while a "guess" judgment was obtained whenever no light at all could be observed by the $S$ or when the localization of the flash was difficult.

There is a further implication to these results. They point out the difficulty of identifying homologous evoked potential components in AEPs obtained under different conditions. Thus, for example, when the amplitude or latency of AEP components is plotted as a function of stimulus luminance (e.g., Vaughan, 1966; Wicke et al, 1964), a considerable shift in wave form has been reported as near-threshold luminances are reached. This shift has sometimes been attributed to a change from photopic to scotopic vision (Vaughan, 1966). It is worth noting, however, that-the observed change is mainly a disappearance of the early components $(80-100 \mathrm{msec})$ and the appearance of a long latency positive wave. In view of the results of this study, it is likely that the change in AEP with low luminances is due to the increased uncertainty the $S$ has about the stimulus as its luminance is decreased. The late positive wave obtained at low luminances would reflect in this case uncertainty resolution by the stimulus rather than shifts in the visual mechanisms involved.

\section{REFERENCES}

CHAPMAN, R. M., \& BRAGDON, H. R. Evoked responses to numerical and non-numerical stimuli while problem solving. Nature (Lond.), 1964, 203, $1155-1157$

DONCHIN, E., \& COHEN, L. Average evoked potentials and intramodality selective attention. EEG clin. Neurophysiol., 1967, 22, 537-546.

SUTTON, S., TUETING, P., ZUBIN, J., \& JOHN, E. R. Information delivery and the sensory evoked potential. Science, 1967, 155, 1436-1438.

VAUGHAN, H. G. The perceptual and physiologic significance of visual evoked responses recorded from scalp in man. In H. M. Burian et al (Eds.), Cinical electroretinography. (3rd Sym.) Oxford: Pergamon Press, 1966. Pp. 203-223.

WICKE, J. D., DONCHIN, E., \& LINDSLEY, D. B. Visual evoked potentials as a function of flash luminance and duration. Science, 1964, 146, 83-85. NOTES

1. The writer is NRC-Resident Research Associate, on leave from the University of Illinois. The data were collected at the laboratory of Dr. D. B. Lindsley at U.C.L.A. with the support of NSF Grant GB 1844 and NASA Contract NsG-623.

2. There were very few instances in which a "guess" report was associated with a "correct" identification. It was thus impossible to obtain an AEP for this condition. 